\title{
July 2013 Critical Care Case of the Month: The Fortuitous Critical Care Consult
}

\author{
Clement U. Singarajah, M.D. \\ Elijah Poulos, M.D. \\ Phoenix VA Medical Center \\ Phoenix, AZ
}

History of Present IIIness

A 70 year old male with squamous cell cancer of the hypopharynx had undergone a laser ablation and debridement as an outpatient. The ENT surgeon placed a \# 6 Shiley DCT tracheostomy tube and the patient did well after the procedure. His chest x-ray after the procedure revealed right lower lobe atelectasis but was interpreted as otherwise normal (Figure 1).

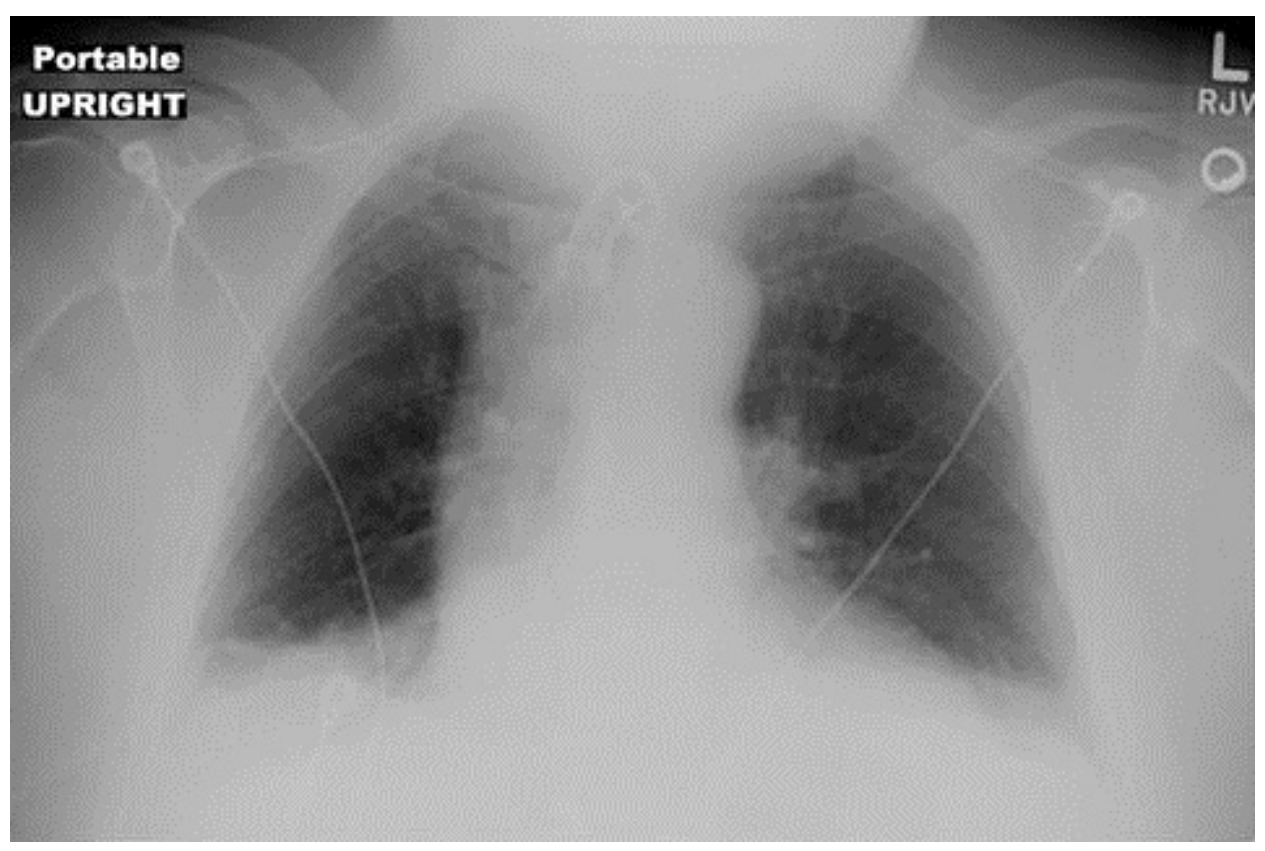

Figure 1. Portable chest-ray after laser ablation and tracheostomy placement.

Due to aspiration and feeding issues, he was scheduled 2 weeks later for percutaneous endoscopic gastrostomy (PEG) tube placement as an outpatient. However, the gastroenterologist cancelled the procedure due to copious secretions from tracheal site, described as purulent and some mild respiratory distress. He was admitted to the general medicine service at the Phoenix VA Medical Center.

\section{Physical Examination}

On examination of the patient, was non-toxic, talking, and alert. Vital signs were within normal limits, but with he had mild dyspnea and moderately thick secretions. A 
tracheostomy tube was in place in the neck. There were no areas of tenderness over his neck. The remainder of his physical examination was normal.

\section{Radiography}

A chest x-ray was performed (Figure 2).
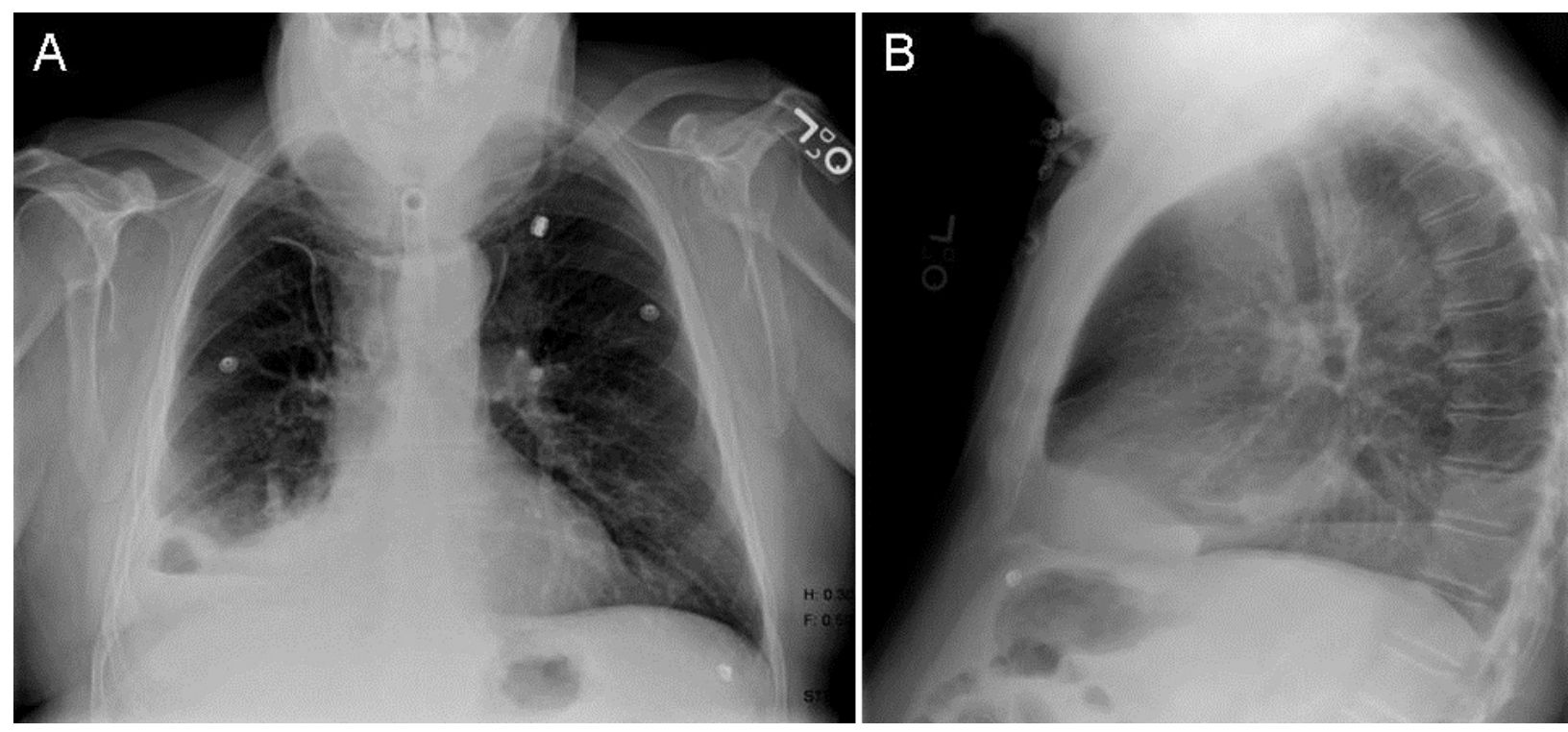

Figure 2. Admission PA (Panel A) and lateral (Panel B) chest X-ray.

Which of the follow are abnormal findings of the chest radiography?

1. The distal tip of the tracheostomy tube is not aligned with the tracheal stripe

2. There is a right pleural effusion

3. There is an air-fluid level in the right lower lung

4. There is right lower lobe atelectasis and/or consolidation

5. All of the above 


\section{Correct! \\ 5. All of the above}

There are several areas of the chest x-ray which are concerning. First, the tip of the tracheostomy tube is not aligned with the tracheal strip (Figure 3). Second, there appears to be dense consolidation and possibly an air fluid level in the right lower lung (Figure 3).
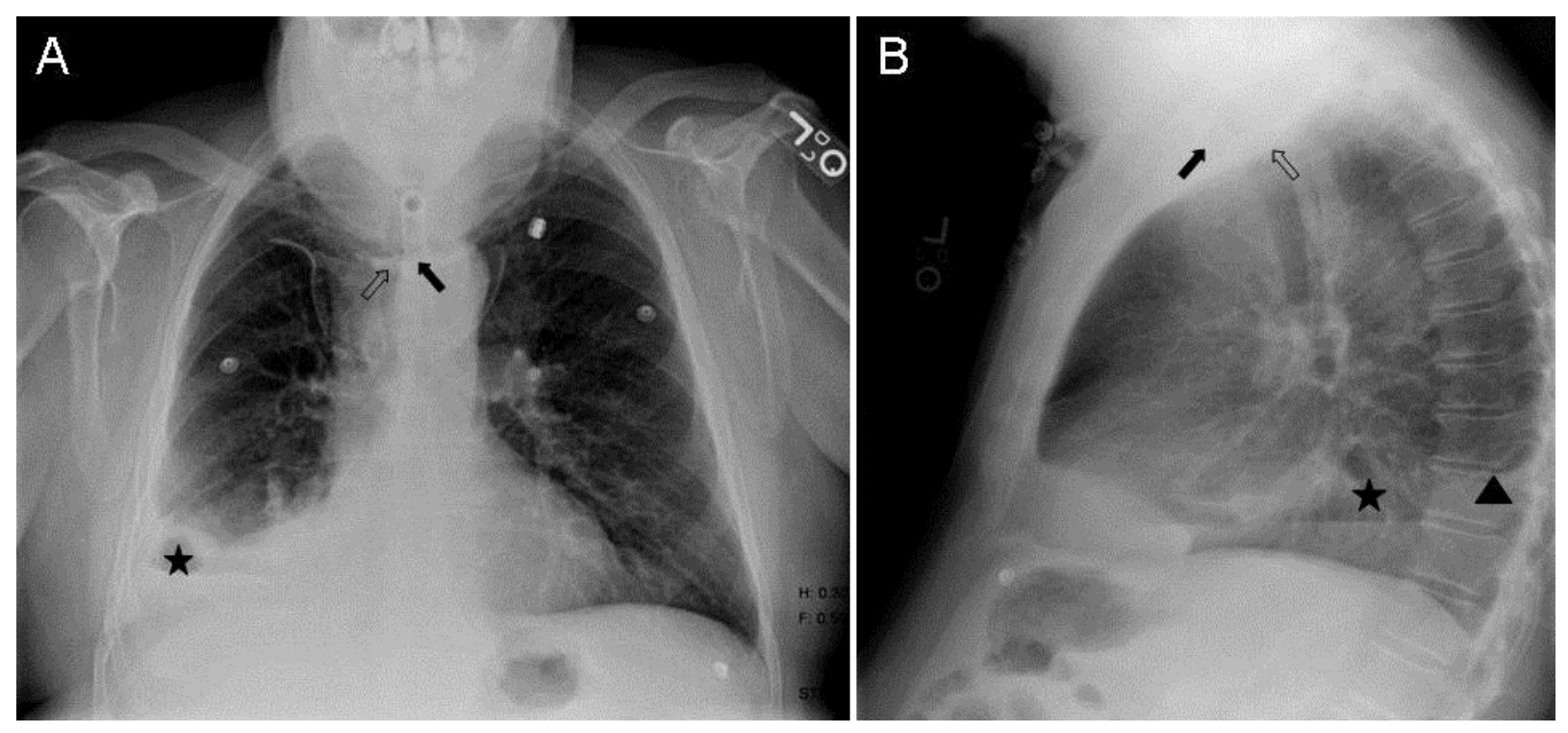

Figure 3. Admission chest x-ray showing the tip of the tracheostomy tube (solid arrow) is not aligned with the tracheal strip (open arrow). There is a question of an air-fluid level in the right lower chest (solid star). The meniscus seen on the lateral (solid triangle) indicates a probable pleural effusion.

Which of the following do the lack of alignment of the tip of the tracheostomy tube and the tracheal stripe and the air fluid level indicate?

1. The air-fluid level can indicate either a pulmonary abcess or an empyema

2. The air-fluid level indicates a pulmonary abcess

3. The lack of alignment of the tip of the tracheostomy tube and the tracheal stripe indicates that the tracheostomy is not in the trachea

4. The lack of alignment of the tip of tracheostomy tube and the tracheal stripe is normal and due to the tube and tracheal stripe being in two different planes

5. 1 and 3

6. 2 and 4 


\section{Correct!}

\section{2 and 4}

The lack of alignment of the distal tip of the tracheostomy tube and the tracheal stripe is disturbing. They should be aligned because they should be in the same plane and so rotation of the patient should not cause the two to be misaligned. This suggests that the tracheostomy tube is not in the trachea.

The air-fluid level is also abnormal. Air-fluid levels can indicate either a lung abcess with air above a cavity containing fluid in the lung or air within the pleural space from an infected pleural space or empyema.

At this juncture a pulmonary/critical care consult was ordered because the ward team thought the patient was in impending respiratory failure for continuous positive airway pressure/noninvasive ventilation (CPAP/NIV).

Which of the following is the next most appropriate step(s) in the management of this patient?

1. Begin high dose penicillin for a pulmonary abcess

2. Insert a thoracostomy (chest) tube

3. Perform a thoracic CT scan

4. Push the tracheostomy tube in deeper

5. All of the above 


\section{Correct! \\ 3. Perform a thoracic CT scan}

Pushing a tracheostomy tube in place unless there is a clear tract to the trachea is hazardous (1). Placing a thoracostomy tube is reasonable if you are sure there is fluid in the pleural space that needs to be drained. Similarly, penicillin is a reasonable choice for a patient who likely has a lung abcess from aspiration and/or an empyema pending culture results. However, the best answer, and the one suggested by the critical care consult, was to perform a CT scan. The thoracic CT should define whether the tracheostomy tube is outside the trachea and whether there is an empyema and/or a lung abcess.

The patient's thoracic CT scan is shown in Figure 4.
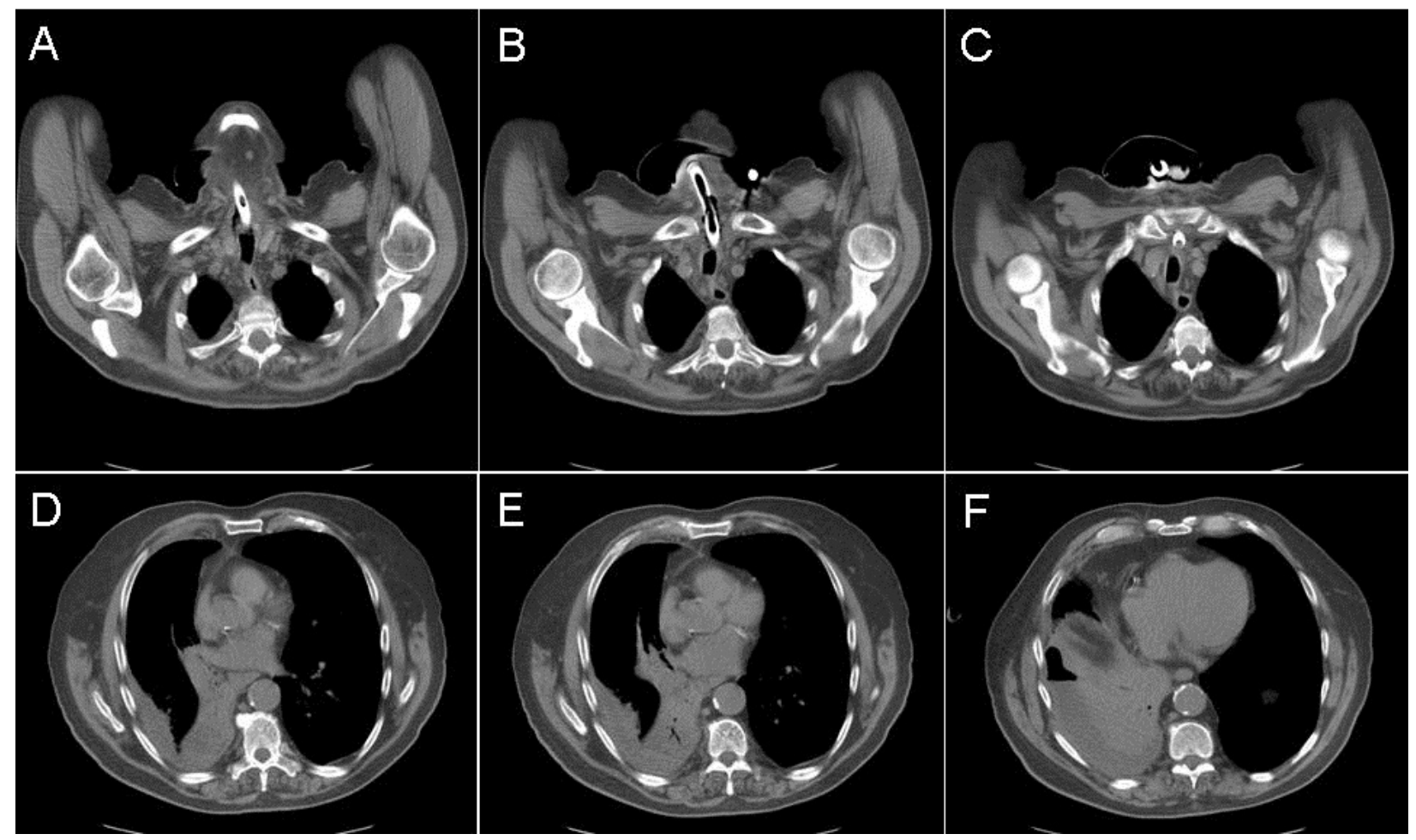

Figure 4. Selected static images from the thoracic CT scan.

Which of the following is not appropriate therapy?

1. Begin CPAP/NIV for impending respiratory failure

2. Begin penicillin for aspiration pneumonia and empyema

3. Insert a thoracostomy tube for a presumptive empyema

4. Replace the tracheostomy tube with a longer tube under bronchoscopic guidance

5. All of the above are appropriate 


\section{Correct!}

\section{Begin CPAPINIV for impending respiratory failure}

The thoracic CT scan shows several problems. First, the tracheostomy is too short in this large man and does not reach the trachea. Second, there is consolidation in the right lower lobe but also clearly a right pleural effusion with an air-fluid level.

Continuous positive airway pressure/noninvasive ventilation (CPAP/NIV) is not appropriate in this patient for several reasons (2). First, CPAP/NIV is relatively contraindicated with copious secretions. CPAP/NIV may push the secretions deeper into the airways and limit secretion mobilization. It may also dry secretions making the secretions thicker and more tenacious. Second, the patient has a relatively focal process. CPAP or other positive airway pressure may over-distend the most compliant portions of the lung which are normal. This may shunt blood to the less compliant portions which have poor ventilation worsening ventilation-perfusion mismatch. Lastly, the tracheostomy tube is not in the trachea and beginning CPAP/NIV will likely result in the air in the mediastinum.

The patient was transferred to the ICU and underwent the placement of a new longer Shiley XLTCP (eXtra Long Cuffed Tracheostomy Proximal) under bronchoscopic guidance. He was begun on mechanical ventilation. A thoracostomy tube was inserted and purulent, odiferous fluid was obtained which grew beta-hemolytic Streptococcus. The patient improved with ampicillin/sulbactam and a brief course of mechanical ventilatory support.

The patient was clinically improving but a repeat thoracic CT scan showed residual fluid. Which of the following has been shown to improve pleural fluid drainage in empyema?
1. DNase
2. Streptokinase
3. Tissue plasminogen activator (t-PA)
4. 1 and 3
5. All of the above 


\section{Correct!}

\section{1 and 3}

Recently, the combination of DNase and tissue plasminogen activator has been shown to improve fluid drainage in patients with pleural infection and reduce the frequency of surgical referral and the duration of the hospital stay (3). Treatment with DNase alone or t-PA alone was ineffective. In the largest randomized trial, the use of streptokinase resulted in no reduction in mortality, decortication rates or hospital days compared with placebo in the treatment of empyema (4).

\section{Pearls}

1. Tracheostomy misadventures can occur more than 2 weeks after a surgical tracheostomy placement and can be difficult to detect clinically.

2. Urgent diagnostic and therapeutic drainage of empyema is critical.

\section{References}

1. Rana S, Pendem S, Pogodzinski MS, Hubmayr RD, Gajic O.Tracheostomy in critically ill patients. Mayo Clin Proc. 2005;80(12):1632-8. [CrossRef] [PubMed]

2. Hess DR. The evidence for noninvasive positive-pressure ventilation in the care of patients in acute respiratory failure: a systematic review of the literature. Respir Care. 2004;49(7):810-29. [PubMed]

3. Rahman NM, Maskell NA, West A, et al. Intrapleural use of tissue plasminogen activator and DNase in pleural infection. N Engl J Med. 2011;365(6):518-26. doi: 10.1056/NEJMoa1012740. [CrossRef] [PubMed]

4. Maskell NA, Davies CW, Nunn AJ et al. U.K. Controlled trial of intrapleural streptokinase for pleural infection. N Engl J Med. 2005;352:865-74. [CrossRef] [PubMed] 